Alma Mater Studiorum - Università di Bologna DEPARTMENT OF ECONOMICS

A new dynamic mechanism to the marriage problem with a variant

Bora Evci

Quaderni - Working Paper DSE N973

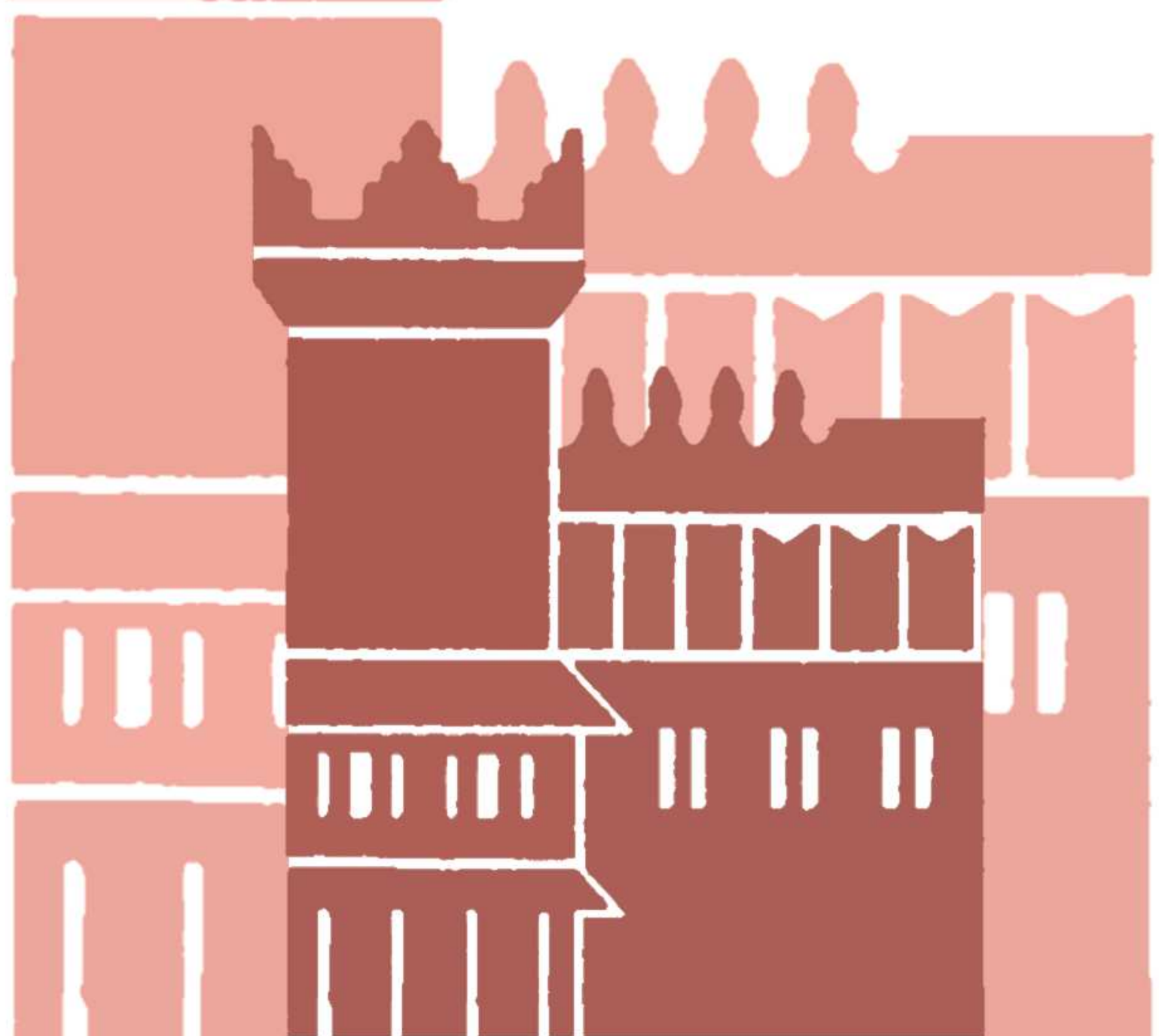




\title{
A NEW DYNAMIC MECHANISM TO THE MARRIAGE PROBLEM WITH A VARIANT*
}

\author{
Bora Evci ${ }^{\dagger}$
}

October 23, 2014

\begin{abstract}
We know from Gale and Shapley (1962) that every Two-Sided Matching Game has a stable solution. It is also well-known that the number of stable matchings increases with the number of agents on both sides.

In this paper, we propose two mechanisms, one of which is a variant of the other, to the marriage problem. Our original mechanism implements the full set of stable matchings for any preference profile. On the other hand, the variant mechanism parititons the domain of preference profiles into two; for one set, it implements the full set of stable matchings like the original mechanism and for the other, it ends up with a proper subset of the set of stable matchings. Besides, for some profiles with multi stability, it gives one of the optimal stable matchings. Namely, the second mechanism coincides either with the original mechanism or it is an improvement for one side; and in some profiles, the algortihm induces Gale and Shapley's algorithm for some profiles. Thus, it is a "middle" mechanism.
\end{abstract}

JEL CODES: C78, D78.

Keywords: Two-Sided Matching Markets, Implementation, Stable Matching, Subgame Perfect Nash Equilibrium.

*I would like to thank Vincenzo Denicolò for his great comments and guidance on the paper. I am grateful to Giacomo Calzolari for his invaluable comments and support from the beggining of the project up to this point. I am indebted to Nadia Burani for the discussions at the beginning of the paper. All errors are my own.

†University of Bologna, Italy. Email: bora.evci3@unibo.it 


\section{Introduction}

After Gale and Shapley (1962) described the well-known marriage problem, a huge literature has accumulated mainly on two-sided matching games. In terms of the interactions between agents of both sides, two types of frameworks have been proposed so far for the matching markets; one is centralized and the other is decentralized. In decentralized market form, the agents match with their opponents on their own. In other words, they are free to match without any third-party interference. As an example, we can give marriages in real life. People meet with each other and decide to get married. In centralized markets, there is a central authority, a clearinghouse or a social planner. This central authority is responsible for the matching process. The agents on boths sides of the market interact with this central agent and submit their preferences. Then, the planner finds by processing the pereferences of all agents via some algorithm and announces the outcome matching. The college admissions in (especially) some eastern countries could be given as an example.

One of the famous examples of centralized markets is the Turkish Student Placements to the universities. High school graduates, who want to be enrolled to a college program, every year take several nation-wide exams organized by the central clearinghouse, ÖSYM, in Turkey. Those exams cover all the subjects in high school curriculums. By assigning different weights to the questions in different subjects, ÖSYM ranks all the students in many different categories, i.e. in category $\mathrm{A}$ the value of a question in Mathematics is 1 point, History $0.8, \ldots$ etc; on the other hand, in category B, Geography 1 point, Chemistry 0.6 point, ....and so on. And, every program type, e.g. Economics, Mathematics, ...etc, belongs to one of those categories, i.e. all economics departments are in category $\mathrm{F}$ and all mathematics departments are in category D. Knowing their ranks in all categories, each student submits a list of a limited number of schools to ÖSYM. (Currently, that number is 30). ÖSYM processes the school lists together with the ranks of the students by running "an algorithm" and announces the placements.

By omitting the resricting assumption on the school lists, Balinski and Sönmez (1999) showed that the algorithm used by ÖSYM in Turkey is equivalent to College-Proposing Gale-Shapley's algorithm, which had been theoretically known as stable. In their paper, they claimed that the algorithm should be converted into Student-Proposing Gale-Shapley for the sake of the students.

Introducing the restriction on the school lists, Doğan and Yuret (2010) showed that the Turkish placement procedure has some inefficiencies. Using data in a fixed year, they showed that the outcome matching of the placements was not stable and they emprically tried to estimate the ratio of the blocking pairs. They stated that the algorithm is equivalent to the one by Gale and Shapley, but because of the restrictions in the application of the algortihm, the procedure generates blocking pairs. They claimed that the limit for the number of schools should be increased to overcome this problem. (At the time of their paper, the limit on the school lists was 18).

Given that the number of agents is too high in this market, the restriction on 
the school lists by the central office is justifiable. Every year nearly two million students take those exams and hundreds of thousands of them are assigned to the universities.

This paper started with the aim to reduce, and eliminate if possible, the inefficiencies of this huge market in Turkey. For this purpose, we proposed a mechanism. Our mechanism partially mimics this Turkish market. In our market form, the role of the colleges is same as with the one in real life; they "submit" their preferences over the students to the clearinghouse. On the other hand, we have changed the game scenario for the students. Instead of submitting their preferences, students choose their partners (school seats) themselves. So, while the game for the schools is centralized, it is decentralized for the students. Thus, we call this market as "semi-centralized".

We illustrate our model through college admission procedure. We call the side of the schools as "the Restricters" and the side of students as "the Choosers". We call the colleges as restricters, because they restrict the game of the other side. And, since the students choose their partners, they are called the choosers.

In our model, the restricters are regarded as objects and their job is done once they submit their preferences. The game is played among the choosers. In the mechanism, the choosers act simultaneously at each rank in the preferences of the restricters. Once a chooser accepts the offer of a resricter, they form a pair and both of them are deleted from the pool. If he rejects the offer and decides to wait his turn for another restricter, then he loses his chance for that restricter forever.

In section 3, we describe an example on how this mechanism solves some of the inefficiency problems of Turkish market in real life, that is under incomplete information. When we introduce complete information to the model, our mechanism implements the full set of stable matchings for any preference profile.

And then, we introduce a minor modification to our mechanism. Basically, when a chooser agent rejects an offer of a restricter, instead of losing him forever, he is replaced to the end of the queue of the same restricter. We show that this modification generates a new "middle" mechanism. Briefly, this new mechanism partitions the full domain of the preference profiles. In one of the set, it again implements the full set of stable matchings and in the other, it implements a proper subset of the set of stable matchings for the profile. Besides, for some profiles it ends up with the Chooser-Optimal stable matching, which could be got by chooser proposing Gale-Shapley's algorithm. Thus, this variant mechanism is partially biased for one side of the market.

Full or partial implementation of stable matchings is not a new issue. In the relevant literature, some mechanisms have been proposed so far rely on different game scenarios (two-stages, multi-stages) for different equilibrium concepts (undominated Nash equilibrium, subgame perfect Nash equilibrium) in different market forms (centralized, decentralized).

Alcalde (1996) proposed a deferred acceptance algorithm similar to GaleShapley, but in a now-or-never scenario. That is, if an agent receives an offer, she can never receive an offer in the subsequent stages. In every period, in the proposing stage each agent proposes to their best choice and the receiver agents 
pick the best offer. Contrary to our mechanism, in Alcalde's mechanism, the receivers are treated as the objects. Alcalde showed that in undominated Nash equilibria, the mechanism ends up with the full set of stable matchings.

Alcalde et al. (1998) proposed a two-stage mechanism. In the first stage, each firm proposes a vector of salaries one for each worker. The firms move simultaneously. Once the salaries have been announced, each worker selects a firm. The decisions in the second stage determine the matching. This mechanism implements the full set of stable matchings in subgame perfect Nash equilibrium.

Alcalde and Romero-Medina (2000) proposed a two-stage game for many-toone games. In the first stage, each student simultaneously sends a letter to at most one college. In the second stage, each college selects its best set of students among their candidates. Colleges' decisions determine the matching. Like in Alcalde(1996), the receivers are the objects of the game. They show that this mechanism implements the full set of stable matchings in subgame perfect Nash equilibrium.

Blum, Roth and Rothblum (1997) proposed a deferred-acceptance process. Their paper extends the theory of two-sided matching models in a way to be applied to senior level and decentralized labor markets. This paper shows how a market may re-gain stability after a stable matching is disrupted. They assume there is uncertainty; each proposer only knows to whom she proposes and each receiver-replier knows only his offers. Also, the order of the proposers to make offers is randomized. They analyzed the Nash equilibria. In their paper, the order of the proposers are randomized and they show that the outcome of the mechanism mainly depends on the initial point of the game. While empty input (no matching) results in a proposer-optimal matching (like Gale and Shapley), starting with a non emtpy (disrupted) matching gives a decider-worst matching, which is better than the initial matching for the deciders. They also show that the decentralized and the centralized equilibria coincide.

Peleg (1997) proposed a one-stage game for the marriage problem. Agents on both sides propose to at most one agent on the other side. If a man and a woman propose each other, then they form a pair. Peleg showed that his mechanism implements the full set of stable matchings by strong Nash equilibria. He also showed that a simple extensive form game finds the same set in subgame perfect Nash equilibrium.

Roth and Xing (1997) proposed a deferred acceptance algortihm for the market for clinical psychologists, which is decentralized. In their paper, the "duration" of the decisions is emphasized. When the agents on one side of the market propose, the other side can hold the offers for a while so that they can wait for better proposals safely. They show that the results coincide with Gale-Shapley's decentralized algortihm.

Diamantoudi et al. (2007) proposed an infinite-horizon decentralized game by extending the original Gale-Shapley model to a dynamic and non-cooperative setting, where firms and workers interact repeatedly over the years. Every period consists of two stages. In the first stage, each firm proposes to at most one worker. In the second stage, active workers, who have no commitment, accept or 
reject the proposals. They studied the effect of commitment in three scenarios, i.e. no commitment, one-sided comitment and two-sided commitment. They found that under no commitment, any stable matching can be yielded. Under two-sided commitment, instability is observed. Under one-sided version, the resulting instability is worse than any other stable matching for workers.

Pais (2008) proposed a multi-period decentralized market. The firms are randomly chosen to make offers. Workers accept or reject the offers. Once rejected, a firm does not propose to the same worker again. If even a worker accepts an offer, he can stay in the market to wait for a better proposal. Firms are also free to fire a worker to propose to some other workers. Hence, any period may start with a matching or an empty matching. Starting with same matching may not need to end up with the same outcome matching. They showed that if the initial matching is empty, every stable matching can be reached as the outcome of an ordinal equilibrium.

Niederle and Yariv (2009) proposed a multi-period mechanism for firmworker one-to-one games. They restrict their attention to the preference profiles with unique stable matchings. Every period, the firms can propose to a worker if the firm has no proposal held by another worker. Workers can accept, reject or hold the offers, in which case the worker is available also in the next period. They concentrate on equilibria in weakly undominated strategies. They showed that under complete information, the game ends up with (the only) stable matching. When the information is incomplete, the property of holding the proposals and uniquness of stability help to end up with stability.

Haeringer and Wooders (2011) proposed a sequential mechanism for decentralized markets and they studied the mechanism for four different scenarios. In their game, the firms propose and the workers accept or reject the offers. Their scenarios are based on whether firms and workers acts simultaneously or non-simultaneously. In their paper, they emphasized the effect of commitment on the ourcome matching. Under commitment, they show that regarless of how the firms play, if the workers act simultaneously, the outcome includes the full set of stable matchings; but it also includes unstable solutions. If they act nonsimultaneously, the result is worker-optimal stable matching. When there is no commitment, the result is always worker-optimal stable matching. Equilibrium concept in this paper is subgame perfect Nash equilibrium.

Romero-Medina and Triossi (2013) proposed an extension of the model by Alcalde and Romero-Medina (2000). Precisely, they extended the serial dictatorship. Students simultaneously propose to the colleges. And, then, the colleges in a fixed order are allowed to accept their offers in one single queue. They show that this extended-serial dictatorship mechanism implements the full set of stable matchings in subgame perfect Nash equilibrium. Here, the proposers' (students) decisions are irreversable. They showed that if the decisions are not irreversable, the unstable matchings could arise in equilibrium.

Among those papers, our paper is based on a semi-centralized framework with a multi-stage game for one side where we find the outcome in subgame perfect Nash equilibrium. In this perspective, it is similar to Romero-Medina and Triossi (2013), but we allow multi-ordering; not restricted to one single 
queue. Another similar paper is Alcalde and Romero-Medina (2000), but their game scenario is based on two stages. There is a similarity between our paper and Alcalde (1998). But, unlike ours, his game scenario is based on a two-stage game; one-stage for each side like Alcalde and Romero-Medina (2000). There is also a similar work to our paper by Haeringer and Wooders (2011) in the sense that there are multi-stages for non-proposers. But, we fix the preferences of one side which makes them non-strategic players, namely "objects" in the game. Their game consists of several two-stage games. But, in our paper, for one side (restricters) it is a one-stage game and for the other side (choosers) it is a multi-stage game and they play the game with each other; not against the restricter agents on the other side. Since our models are different, we observe the differences in the outcomes. Our mechanism never ends up with only an optimal stable matching for any side in the existence of multi stability; it does if and only if there is only one stable matching for the preference profile. Besides, since we work under complete information, our mechanism does not choose any unstable matching in subgame perfect Nash equilibrium. Our model may also seem as an extension of Niederle and Yariv (2009). But, we do not allow the receiver agents to hold an offer. Our analysis also covers the unique stability environments and so we verify their findings on the relationship with the nocycle property and stability uniqueness in our game scenario.

Finally, to our knowledge, having the property to divide the domain of preference profiles into two sets and having the characteristics of two different mehanisms, and so being a middle mechanism between two, our variant mechanism is the first in that sense in the literature. While the mechanism keeps the symmetry between the sides of the market for some preference profiles, it generates a bias for a prefered side for some profiles. Thus, it is a partially biased mechanism.

The paper is organized as follows. Section 2 summarizes some preliminary notes. In Section 3, we present our mechanism and make an exhaustive analysis of the game scenarios. In Section 4, we introduce our variant mechanism and several examples to show the differences from the original mechanism. Section 5 concludes.

\section{Basic Definitions and Notations}

Let $M=\left\{m_{1}, \ldots, m_{k}\right\}$ and $W=\left\{w_{1}, \ldots, w_{l}\right\}$ be two non-empty, finite and disjoint sets of agents (e.g. men and women).

Each agent has a strict preference ordering $R$ over the agents of the other set; for example $R_{m_{i}}$ is the preference ordering of $m_{i} \in M$ over $W$. For any $w_{i}, w_{j} \in W, w_{i} R_{m_{i}} w_{j}$ means $m_{i}$ prefers $w_{i}$ over $w_{j}$. A Preference Profile $\mathbb{R}=R^{M \cup W}=\left(R_{i}\right)_{i \in M \cup W}$ is a set of preference orderings, one for each agent in the model. Let $\Re$ be the set of all preference profiles.

$r_{w_{j}}\left(m_{i}\right)$ is the rank of agent $m_{i} \in M$ in the preference ordering of agent $w_{j} \in W$. For example, $r_{w_{j}}\left(m_{i}\right)=k$ means that $m_{i}$ is the $k^{t h}$ best man for $w_{j}$.

A (two-sided) matching $\mu: M \cup W \rightarrow M \cup W$ is an injection. For any 
$m_{i} \in M$ and $w_{j} \in W, \mu\left(m_{i}\right)=w_{j}$ means that $w_{j}$ is the match of $m_{i}$ and vice versa. $\mu\left(m_{i}\right)=m_{i}$ means that $m_{i}$ is single in the matching $\mu$. $\Pi^{M \cup W}$ is the set of all matchings between $M$ and $W$.

Let $\mu_{x}, \mu_{y} \in \Pi^{M \cup W}$ be two matchings and $m_{i} \in M$. We can rank matchings, from the point view of agent $m_{i}$, according to how $m_{i}$ ranks the agents he is macthed with. If $\mu_{x}\left(m_{i}\right) R_{m_{i}} \mu_{y}\left(m_{i}\right)$, then we say that for agent $m_{i}, \mu_{x}$ Pareto Dominates $\mu_{y}$. If $\mu_{x}\left(m_{i}\right)=\mu_{y}\left(m_{i}\right)$, then $m_{i}$ is indifferent between $\mu_{x}$ and $\mu_{y}$ and we denote this by $\mu_{x} I_{m_{i}} \mu_{y}$. If $\nexists m_{i} \in M$ such that $\mu_{y}\left(m_{i}\right) R_{m_{i}} \mu_{x}\left(m_{i}\right)$ and $\exists m_{j} \in M$ such that $\mu_{x}\left(m_{j}\right) R_{m_{j}} \mu_{y}\left(m_{j}\right)$, then we say that for the set of men $M, \mu_{x}$ Pareto Dominates $\mu_{y}$; that is $\mu_{x} R_{M} \mu_{y}$. If $\exists m_{i} \in M$ such that $\mu_{y}\left(m_{i}\right) R_{m_{i}} \mu_{x}\left(m_{i}\right)$ and $\exists m_{j} \in M$ such that $\mu_{x}\left(m_{j}\right) R_{m_{j}} \mu_{y}\left(m_{j}\right)$, then we say that for the set of men $M, \mu_{x}$ and $\mu_{y}$ are incomparable.

For any preference profile $\mathbb{R}=R^{M \cup W}=\left(R_{i}\right)_{i \in M \cup W}$ and a matching $\mu$, for any $m_{i} \in M$ and $w_{j} \in W,\left(m_{i}, w_{j}\right) \notin \mu$ is called a blocking pair, if $w_{j} R_{m_{i}} \mu\left(m_{i}\right)$ and $m_{i} R_{w_{j}} \mu\left(w_{j}\right)$. If there is no blocking pair for $\mu$, then we say $\mu$ is stable; otherwise, it is unstable.

Gale and Shapley (1962) proved that for any two-sided matching game $\mathbb{R}=$ $\left(R_{i}\right)_{i \in M \cup W}$, there exists a matching $\mu \in \Pi^{M \cup W}$ which is stable for $\mathbb{R}$.

A Matching Mechanism $\gamma$ is a procedure to select a matching from every preference profile. Formally

$$
\gamma: \Re \longrightarrow \Pi^{M \cup W}
$$

A Matching Mechanism $\gamma$ is called stable, if it always selects a stable matching.

\section{The Dynamic Mechanism}

In this section, we propose a new dynamic mehanism to the marriage problem. With that mechanism, any stable matching could be chosen for any preference profile.

For a given matching game $\mathbb{R}=\left(R_{i}\right)_{i \in M \cup W}$, we choose one side as the Restricter, and the other side as the Chooser. We use the restricters' preferences as the restrictions or the priorities on the chooser side. The choosers play a game in a ranking order that the restricter side determine. In that game, the information is complete; that is the rule of the game and the preference profile is known by all agents. The mechanism could be applied also to incomplete information cases. Here is how the mechanism works.

Without loss of generality, we assign $M$ as the restricter and $W$ as the chooser sides throughout the paper. (Later we will show that set of the outcomes does not depend on which set is the restricter or the chooser).

We start with the woman/women who are the best in view of some men; that is we start with women such that $\left\{w_{i} \in W \mid \exists m_{j} \in M\right.$ such that $\left.r_{m_{j}}\left(w_{i}\right)=1\right\}$. Those women are called to make a decision; either to say "yes" or "no" to men for whom they are the best women. Some of them may be the best woman 
for more than one man. In this case, such a woman is asked to choose one of those men. If a woman says "yes" to a man, then they form a pair and both of them are deleted from the profile; if she says "no", she loses that man/men forever and waits for her turn for other men. At the end of the first step, all the choosers, women, are informed about the results/decisions of the step.

At any step/rank $k$, a woman either chooses a man to marry or refuses and waits for her turn for another man. In that way, we construct our pairs.

First, we shall show that this process produces a matching from any profile. Let $w_{i} \in W$ be a chooser agent. At any step where she is the best woman for any man, if $w_{i}$ decides to accept the offer of an agent $m_{j} \in M, w_{i}$ is deleted from the profile and she forms the pair $\left(w_{i}, m_{j}\right)$. If she never never accepts an offer at any step, then she forms the pair $\left(w_{i}, w_{i}\right)$. As we have said before, any chooser says "no" to wait for her turn for a better restricter. In this model, we explicitely assume that all the agents are acceptable for the agents on the other side, and so they prefer being matched to some agent of the other side of the matket rather than being single. If she never says "yes" to any man, she remains single which contradicts to the rationality assumption. We will analyze when and why a woman says "no" in the following sections. If a chooser agent remains single, it is only because she does not receive any offer (when $k<l$ if $w_{i} \in W$ is not a favorite woman). These scenarios are the same for all $w_{j} \in W$. On the other hand, when any $w_{j} \in W$ chooses an agent $m_{i} \in M$, he is deleted from the profile, too. If $m_{i}$ is not choosen by any $w_{j}$ (when $l<k$ if $m_{i}$ is not a favorite man), then he forms the pair $\left(m_{i}, m_{i}\right)$. This happens when all of women are matched to some men before he calls for her "best(s)". So, any agent $i \in M \cup W$ could be a member of one pair. Hence, the outcome of the procedure $\gamma$ is a matching $\mu \in \Pi^{M \cup W}$.

Now, we shall demostrate our mechanism with a simple example.

Example 1 Let $M=\left\{m_{1}, m_{2}, m_{3}\right\}$ and $W=\left\{w_{1}, w_{2}, w_{3}\right\}$ be two disjoint sets who have the following preference profile $\mathbb{R}_{1}$.

$$
\mathbb{R}_{1}=\begin{array}{cccccc}
\frac{m_{1}}{w_{2}} & \frac{m_{2}}{w_{1}} & \frac{m_{3}}{w_{2}} & \frac{w_{1}}{m_{1}} & \frac{w_{2}}{m_{3}} & \frac{w_{3}}{m_{1}} \\
w_{1} & w_{3} & w_{3} & m_{3} & m_{2} & m_{2} \\
w_{3} & w_{2} & w_{1} & m_{2} & m_{1} & m_{3}
\end{array}
$$

In the first round, $w_{1}$ and $w_{2}$ are asked to choose; $w_{1}$ for $m_{2}$ and $w_{2}$ for either $m_{1}$ or $m_{3}$. Since $r_{w_{2}}\left(m_{3}\right)=1, w_{2}$ says "yes" to $m_{3}$. They construct $\left(m_{3}, w_{2}\right)$ and both of them are deleted from the profile. As the information is complete and so $w_{1}$ knows that $w_{2}$ prefers $m_{3}$ over $m_{1}$, she says "no" to $m_{2}$.

In the second round, $w_{1}$ and $w_{3}$ are asked to choose; $w_{1}$ for $m_{1}$ and $w_{3}$ for $m_{2}$. Since $r_{w_{1}}\left(m_{1}\right)=1, w_{1}$ says "yes" to $m_{1}$. They construct $\left(m_{1}, w_{1}\right)$ and both of them are deleted from the profile. Eventhough $r_{w_{3}}\left(m_{1}\right)=1$, since the information is complete and so $w_{3}$ knows that $w_{1}$ chooses $m_{1}$, she says "yes" to $m_{2}$.

Hence, using our mechanism $\gamma$ we get the matching $\mu=\left\{\left(m_{1}, w_{1}\right),\left(m_{2}, w_{3}\right),\left(m_{3}, w_{2}\right)\right\}$ which is the only stable matching for $\mathbb{R}_{1}$. 
Before we analyze the details of the flow of the game under complete information, we shall present a real life example to show that this mechanism is also successful under incomplete information. Assume $s$ is a student in Turkish college admission procedure. Currently, s is allowed to submit at most 30 schools to the central clearing house. He wants to study Economics and there are more than 100 Economics departments in Turkey. By assumption, student $s$ has a preference ordering over the set of all Economics departments.

In the current placement procedure, the school lists are submitted simultaneously and no student has any idea about the preference orderings of any other student. Thus, $s$ should choose his schools very carefully; either he may not be placed to any school if the students who have better rankings than $s$ fill the departments for which $s$ submits, or he may be enrolled to a school worse than some other he could get. And, any of those scenarios generates instability. Thus, the problem is that which schools should $s$ submit.

The mechanism we propose here solves the problem of $s$. Even though he cannot observe the preference orderings of any other student, he is able to observe the taken schools (seats) before his turn comes. When it is his turn, he is perfectly aware of the currently available positions and he chooses the best school among them. In such a case, student $s$ is never a member of a blocking pair. Hence, our mechanism solves some instability problems for some preference profiles. It may not solve inefficiency problems (under incomplete information) for some preference profiles where a student is interested in more than one program type and his turn for a worse type comes before the better one, which we will analyze in the following section.

\subsection{The Flow of the Mechanism}

In this section, we will exhausatively examine the flow of the game. All the possible game scenarios that the choosers confront are based on three definitions we give below.

Definition 2 Let $w \in W$ be any chooser agent and $m_{i}, m_{j} \in M$ be any two restricter agents with $r_{m_{i}}(w)>r_{m_{j}}(w)$ and $w_{i} R_{m} w_{j}$. If at the step $k=r_{m_{j}}(w)$ non of $m_{i}$ and $m_{j}$ have been taken by other choosers yet, then we say the agent $w$ experiences a conflict between agents $m_{i}$ and $m_{j}$.

The definition says that for a chooser agent if the turn for a worse restricter comes before any better one, given that non of those restricters have not been taken yet, then the chooser agent experiences a conflict; he may not be sure about his decision, even under complete information.

Definition 3 If a chooser agent $w \in W$ does not experience any conflict, then we say $w$ has a smooth game.

In this paper, we pay our attention to the special case of conflicts.

Definition 4 Let $\left\{m_{1}, \ldots, m_{r}\right\} \subset M$ be a set of restricters and $\left\{w_{1}, \ldots, w_{r}\right\} \subset W$ be a set of choosers. If we have such a case; 
- $w_{1} R_{m_{1}} w_{2}, w_{2} R_{m_{2}} w_{3}, \ldots, w_{r} R_{m_{r}} w_{1}$,

- $m_{1} R_{w_{2}} m_{2}, \ldots, m_{r-1} R_{w_{r}} m_{r}, m_{r} R_{w_{1}} m_{1}$,

- $r_{m_{1}}\left(w_{1}\right)=r_{m_{2}}\left(w_{2}\right)=\ldots=r_{m_{r}}\left(w_{r}\right)=k$ (for at least one side),

- Each agent of $\left\{w_{1}, \ldots, w_{r}\right\}$ and $\left\{m_{1}, \ldots, m_{r}\right\}$ is present at step $k$.

Then, we say that agents in $\left\{w_{1}, \ldots, w_{r}\right\}$ experience a cyclical conflict with each other for the set $\left\{m_{1}, \ldots, m_{r}\right\}$ at step $k$.

Therefore, even though the preference profile is publicly known, if some group of choosers experience a cyclical conflict, they cannot have a precise decisions, since the actions are not observable for the current step.

Blair (1988) showed that the set of stable matchings has the structure of a partial order for any preference profile. Together with Gale and Shapley (1962) and Knuth (1976), for a preference profile we know that;

i) If there is a unique stable matching, it is men and women optimal stable matching.

ii) If there are two stable matchings, then one of them is men-optimal and the other is women-optimal stable matchings.

iii) If there are three stable matchings, one of them is men-optimal; one another is women-optimal; and the last is a middle stable matching.

$i v$ ) If there are more than three stable matchings, then we have a partial order for both of the sides.

In the following pages of this section, we will construct the full structure of the set of stable matchings through Claim 5 to Theorem 13 based on the game scenarios in above definitions.

Claim 5 At any step $k$, any chooser agent can be a member of at most one cyclical conflict. In other words, he cannot have cyclical conflicts with two disjoint sets of choosers for two disjoint restricters.

Proof. The proof is straightforward. Let $w_{i} \in W$ be any chooser being a member of more than one cyclical conflict at step $k$. Let $m_{i}, m_{j} \in M$ be any two restricters where $r_{m_{i}}\left(w_{i}\right)=r_{m_{j}}\left(w_{i}\right)=k$ and each of these restricters is from different cyclical conflicts that $w_{i}$ experiences. By assumption, all the agents have strict preferences. So, we have either $m_{i} R_{w_{i}} m_{j}$ or $m_{j} R_{w_{i}} m_{i}$. Thus, $w_{i}$ only considers the cycle with the better restricter among $m_{i}$ and $m_{j}$ and he does not consider the worse agent.

Now, we will focus on the effects of such cycles on the relationship between any preference profile and the set of the stable matchings for that profile; ex. the number of stable matchings for a given profile. Since this is the environment that they work on, Niederle and Yariv (2009) states the following theorem (as a proposition) in their paper. Here, we show this result under our game scenario.

Theorem 6 For any given preference profile $\mathbb{R}=\left(R_{i}\right)_{i \in M \cup W}$, there exists only a unique stable matching if and only if there exists no cyclical conflict for the choosers. 
Proof. $(\Longleftarrow)$. Suppose that there is no cyclical conflict. We shall assume multi stability for a preference profile and prove that this leads to a contradiction. We know from Gale and Shapley that there exist optimum stable matchings $\mu_{M}$ and $\mu_{W}$ for men and women, respectively, with $\mu_{M} R_{M} \mu_{W}$ and $\mu_{W} R_{W} \mu_{M}$. Since the preferences are strict, then $\exists w_{k}, w_{l} \in W$ and $\exists m_{i}, m_{j} \in M$, such that

1. $\mu_{M}\left(m_{i}\right)=w_{k}, \mu_{M}\left(m_{j}\right)=w_{l}, \mu_{W}\left(m_{i}\right)=w_{l}, \mu_{W}\left(m^{*}\right)=w_{k}$ and $\mu_{W}\left(m_{j}\right)=$ $w^{*}$ for some $m^{*} \in M, w^{*} \in W$ with

2. $m^{*} R_{w_{k}} m_{i}, m_{i} R_{w_{l}} m_{j}, w_{k} R_{m_{i}} w_{l}, w_{l} R_{m_{j}} w^{*}$.

In that case, we may have two scenarios:

Scenario 1: Let $A=\left\{\bar{m} \in M \mid \bar{m} R_{w_{l}} m_{i}\right\}$ be the set of agents who are better than agent $m_{i}$ according to agent $w_{l}$. Since $\mu_{M}\left(m_{i}\right)=w_{l}$, either all of $\bar{m} \in A$ have already been taken by some other $\hat{w} \neq w_{l}$ before the round where $w_{l}$ chooses $m_{i}$, or else $w_{l}$ could foresee that each $\bar{m}$ would be taken before her turn. Hence, any $\bar{m} \in A$ is not achievable for $w_{l}$. On the other hand, we have $\mu_{W}\left(m_{j}\right)=w_{l}$. In that case, the fact that $m_{i} R_{w_{l}} m_{j}$ contradicts the rationality of agent $w_{l}$; while she could choose $m_{i}$, she did not. This implies that $m_{i}=m_{j}$, which gives $\mu_{M}=\mu_{W}=\mu$, contradiction to the multi stability.

Scenario 2: Maintaining the assumptions on the rationality of agents and multi stability, we have the following scenario. If we have stable matchings $\mu_{M}$ and $\mu_{W}$, using information in 2 above, we may have either of the followings;

1. $w^{*}=w_{k}$ and $m^{*}=m_{j}$, that is the set $\left\{w_{l}, w_{k}\right\}$ has a cyclical conflict for the set $\left\{m_{i}, m_{j}\right\}$ so that the preference profile has such two stable matchings, or

2. $w^{*}=w^{\prime}$ and $m^{*}=m^{\prime}$, that is there is a bigger cycle including $w_{l}, w_{k}, m_{i}$ and $m_{j}$. By iterative construction, we end up with the full cycle of all agents.

Both of them contradict to the fact that there is no cycle. Hence, there is only one stable matching.

$(\Longrightarrow)$. For a profile $\mathbb{R}=\left(R_{i}\right)_{i \in M \cup W}$, there exists only one stable matching $\mu \in \Pi^{M \cup W}$. And, let us assume there exists a cyclical conflict between the agents of $W^{\prime}=\left\{w_{1}, \ldots, w_{r}\right\} \subset W$ for the agents $M^{\prime}=\left\{m_{1}, \ldots, m_{r}\right\} \subset M$ at step $k$.

For each $w_{i} \in W^{\prime}$ is in the cycle, any better restricters than the ones in the cycle have either been taken or regarded as will be taken. For that reason, in the matching $\mu$ we cannot have any pair such that $\left(m_{i}, \hat{w}\right)$ where $m_{i} \in M^{\prime}$ and $\hat{w} \notin W^{\prime}$; because $\left(m_{i}, w_{j}\right)$ would block the matching $\mu$, for some $w_{j} \in W^{\prime}$. For that reason, in the matching $\mu, \forall m_{i} \in M^{\prime}$ and $\forall w_{j} \in W^{\prime}, \mu\left(m_{i}\right) \in W^{\prime}$ and $\mu\left(w_{j}\right) \in M^{\prime}$.

We shall assume that all choosers $w_{j} \in W^{\prime}$ say "yes" at step $k$. In such a case, the matching $\mu_{x}$ would be stable since no pair blocks it; that is $\forall m_{i} \in M^{\prime}$ get better choosers in their own cycle, so no man admires any woman in the 
cycle. But, in that case, another matching $\mu_{y}$ would be stable where no agent $w \in W^{\prime}$ says "yes" at step $k$; in that situation $\forall w_{j} \in W^{\prime}, \mu_{y}\left(w_{j}\right) R_{w_{j}} \mu_{x}\left(w_{j}\right)$. Hence, in $\mu_{y}, \forall w_{j} \in W^{\prime}$ get their better restricters in their own cycle, so no woman admires any man in the cycle. Hence, we have two stable matchings which contradicts the single stable matching assumption. And, this argument is same for any other cyclical conflicts.

With Theorem 6, we have showed that when there is a unique stable matching for a preference profile, we do not have any cyclical conflict for the choosers, and vice versa. Our proof also showed that when there is a unique stable matching, our mechanism finds that matching. As we have a two-sided implication in the theorem, we get a symmetry between both sides: if one side has no cyclical conflict, then there is a unique stable matching and if so, other side has no cyclical conflict. Then, in any scenario, we get the same matching.

Theorem 6 says that the reason for multi stability is the existence of cyclical conflicts. The following example shows that when there are multiple stable matchings for a given preference profile, if we change the roles, the set of agents having cyclical conflicts need not be the same on both sides.

Example 7 Let $M=\left\{m_{1}, m_{2}, m_{3}\right\}$ and $W=\left\{w_{1}, w_{2}, w_{3}\right\}$ be the sets of men and woman, with the following preference profile $\mathbb{R}_{2}$ :

$$
\mathbb{R}_{2}=\begin{array}{llllll}
\frac{m_{1}}{w_{1}} & \frac{m_{2}}{w_{2}} & \frac{m_{3}}{w_{2}} & \frac{w_{1}}{m_{3}} & \frac{w_{2}}{m_{1}} & \frac{w_{3}}{m_{3}} \\
w_{3} & w_{1} & w_{3} & m_{2} & m_{2} & m_{1} \\
w_{2} & w_{3} & w_{1} & m_{1} & m_{3} & m_{2}
\end{array}
$$

There are two stable matchings for $\mathbb{R}_{2}$. When $W$ is the chooser side, the sets of agents in the cycle are $\left\{m_{1}, m_{2}\right\}$ and $\left\{w_{1}, w_{2}\right\}$. On the other hand, when $M$ is the chooser side, the sets of agents in the cycle are $\left\{m_{1}, m_{3}\right\}$ and $\left\{w_{2}, w_{3}\right\}$.

\begin{tabular}{|c|c|c|c|c|c|c|c|c|c|c|c|}
\hline \multicolumn{6}{|c|}{$W$-Chooser case } & \multicolumn{6}{|c|}{ M-Chooser case } \\
\hline$n_{1}$ & $\underline{m_{2}}$ & $\underline{m_{3}}$ & $\underline{w_{1}}$ & $w_{2}$ & $w_{3}$ & $\underline{m_{1}}$ & $\underline{m_{2}}$ & $\underline{m}_{3}$ & $w_{1}$ & $\underline{w_{2}}$ & $w_{3}$ \\
\hline$N_{1}$ & $\mathbf{w}_{2}$ & $w_{2}$ & $m_{3}$ & $\mathbf{m}_{1}$ & $m_{3}$ & $w_{1}$ & $w_{2}$ & $\mathbf{w}_{2}$ & $m_{3}$ & $\mathbf{m}_{1}$ & $\mathbf{1}_{3}$ \\
\hline$\omega_{3}$ & $\mathbf{w}_{1}$ & $w_{3}$ & $\mathbf{m}_{2}$ & $\mathbf{m}_{2}$ & $m_{1}$ & $\mathbf{w}_{3}$ & $w_{1}$ & $\mathbf{w}_{3}$ & $m_{2}$ & $m_{2}$ & $\mathbf{1}_{1}$ \\
\hline$N_{2}$ & $w_{3}$ & $w_{1}$ & $\mathbf{m}_{1}$ & $m_{3}$ & $m_{2}$ & $\mathbf{w}_{2}$ & $w_{3}$ & $w_{1}$ & $m_{1}$ & $\mathbf{m}_{3}$ & $m_{2}$ \\
\hline
\end{tabular}

We have seen that the existence of a cyclical conflict generates two stable matchings; one of them is constructed if all the choosers in the cycle say "yes" in the first step and the second is created if all say "no". But, we cannot conclude that every cycle generates two stable matchings.

Definition 8 Let $M$ and $W$ be the sets of restricters and choosers, respectively. Let $W_{1}, W_{2} \subset W$ be the set of the agents of two cycles. If $W_{1} \cap W_{2}=\emptyset$, then we say that the cycles are independent. Otherwise, they are (inter) dependent.

From Claim 5, we know that any chooser agent cannot have cyclical conflicts with two disjoint sets of choosers for two disjoint restricters at the same step of the game; but he can be a member of another cycle at the same step step of the game. In such a case, that restricter and the relative chooser are the common agents of those two cycles. 
Proposition 9 Two (inter) dependent cycles generate three stable matchings.

Proof. Let $M_{1}, M_{2} \subset M$ and $W_{1}, W_{2} \subset W$ with $M_{1}$ and $W_{1}$ be the agents of the cycle at step $k$ and $M_{2}$ and $W_{2}$ be the agents of the cycle at step $l$. For (inter) dependent cycles, we have two possible scenarios; either $k=l$ or $k \neq l$.

Let us assume that for $W_{1}$ if all say "yes" at step $k, \mu_{1}$ is generated; if nobody says "yes", $\mu_{2}$ is generated. And, also we shall assume that for $W_{2}$ if all say "yes" at step $l, \mu_{3}$ is generated; if nobody says "yes", $\mu_{4}$ is generated. Let $w \subset W_{1} \cap W_{2}$ be a chooser agent of the both of the cycles.

The scenario $k=l$ is trivial. We shall analyze the case $k \neq l$. Let $m_{i} \in M_{1}$ and $m_{j} \in M_{2}$ such that $r_{m_{i}}(w)=k$ and $r_{m_{j}}(w)=l$ with (wolg) $k<l$. If $w$ (with all other agents in $W_{1}$ ) says "no", they come to the cycle $M_{1}-W_{1}$ 's consecutive step to $l$ (here we explicitely assume $l-k \geq 1$ ). If $w$ says "yes" (with all other agents in $W_{1}$ ) at step $l, \mu_{2}$ is generated. But, this also means that $w$ says "yes" (with all other agents in $W_{2}$ ) at step $l$, which is the first step of the cycle $M_{2}-W_{2}$, for which $\mu_{3}$ is generated. Then $\mu_{2}=\mu_{3}$.

The same argument works for the case $k=l$ from which we end up with $\mu_{1}=\mu_{3}$. Hence, two (inter) dependent cycles generate three stable matchings.

From Proposition 9, the idea saying that "each cycle produces two stable matchings" fails. We refer to the following preference profile $\mathbb{R}_{3}$ where $k \neq l$ and $w_{1}$ is the common agent.

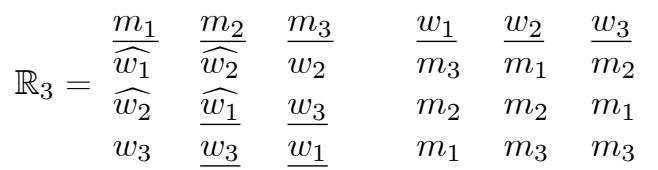

For example, we may have four stable matchings from either two independent cycles or three sequentially (inter) dependent cycles. Hence, there seems to be no clear relationship between the number of cycles and the number of stable matchings for a given profile $\mathbb{R}$.

One more property of cyclical conflicts relates to the set of stable matchings.

Theorem 10 For any preference profile, there exist independent cyclical conflicts which occur at the same step $k$ if and only there exist incomparable stable matchings.

Proof. $(\Longrightarrow)$.Let $M$ and $W$ be the sets of restricters and choosers, respectively, and let $M_{1}, M_{2} \subset M$ and $W_{1}, W_{2} \subset W$ with $M_{1}$ and $W_{1}$ be the agents of the one of the cycles and $M_{2}$ and $W_{2}$ be the agents of the other cycle.

We shall focus on the scenarios of those two cycles. Let us assume that at step $k$, if the agents of $M_{1}$ say "yes", they construct the couples $C 1$, whereas if nobody says "yes", they construct the couples $C 2$. The same argument applies to the agents of $M_{2}$ and they construct the couples $C 3$ from "yes" at step $k$ and $C 4$ from "no" at step $k$. Since the cycles are independent from each other, then the couples constructed by the two cycles are different. The combinations of those cycles give us four matchings: $C 1 \cup C 3 \in \mu_{1}, C 1 \cup C 4 \in \mu_{2}, C 2 \cup C 3 \in \mu_{3}$ and $C 2 \cup C 4 \in \mu_{4}$. From the definition of a cycle, we have 


\section{1. $C 2 R_{M_{1}} C 1$ and $C 4 R_{M_{2}} C 3$,}

2. $C 1 R_{W_{1}} C 2$ and $C 3 R_{W_{2}} C 4$.

Hence, from (1) we have $\mu_{1} R_{W} \mu_{4}$ and $\mu_{4} R_{M} \mu_{1}$. And, from (2), we find that $\mu_{2}$ and $\mu_{3}$ are incomparable for both sides $M$ and $W$.

$(\Longleftarrow)$. The proof of this direction is straight forward. Let $\mu_{x}$ and $\mu_{y}$ be any two incomparable stable matchings for the profile $\mathbb{R}=\left(R_{i}\right)_{i \in M \cup W}$ with $M$ be the restricter and $W$ be the chooser sides. From Theorem 6 , we know that the reason of multi stability is the existence of cyclical conflicts. Since $\mu_{x}$ and $\mu_{y}$ are incomparable stable matchings, there are two sets $M_{1}$ and $M_{2}$ such that $M_{1}=\left\{m_{i} \mid \mu_{y}\left(m_{i}\right) R_{m_{i}} \mu_{x}\left(m_{i}\right)\right\}$ and $M_{2}=\left\{m_{j} \mid \mu_{x}\left(m_{j}\right) R_{m_{j}} \mu_{y}\left(m_{j}\right)\right\}$. Since the interests of the agents in $M_{1}$ and $M_{2}$ are opposite, it is easy to show that they are constructed from different cycles. If they were sequential cycles, i.e. one of them comes at an earlier step of the game, there would not be any conflicts between agents on the matchings, since the common benefits of a side (the restricters in our analysis) decreases as we proceed on the rankings from top to bottom. Hence, the cycles should occur at the same step. And, moreover, while one of the matchings is generated from the first step of one of the cycles and the second step of the other, for the other matching it is the opposite.

Now we shall give an example of incomparable stable matchings.

Example 11 (Roth and Sotomayor (1990), Example 2.17, page 37). Let $M=$ $\left\{m_{1}, m_{2}, m_{3}, m_{4}\right\}$ and $W=\left\{w_{1}, w_{2}, w_{3}, w_{4}\right\}$ be the sets of men as the restricter and women as the chooser sides who have the following preference profile $\mathbb{R}_{4}$ :

$$
\mathbb{R}_{4}=\begin{array}{cccccccc}
\frac{m_{1}}{w_{1}} & \frac{m_{2}}{w_{2}} & \frac{m_{3}}{w_{3}} & \frac{m_{4}}{w_{4}} & \frac{w_{1}}{w_{4}} & \frac{w_{2}}{w_{3}} & \frac{w_{3}}{\widetilde{m_{2}}} & \frac{w_{4}}{\widetilde{m_{1}}} \\
\widetilde{w_{3}} & w_{4} & w_{3} & \widehat{w_{3}} & \widehat{w_{4}} & \widehat{w_{1}} & \widehat{m_{2}} \\
\widetilde{w_{4}} & \widehat{w_{3}} & \widehat{w_{2}} & \widehat{w_{1}} & m_{2} & m_{1} & m_{4} & m_{3} \\
m_{3} & m_{4}
\end{array}
$$

$\Pi^{M \cup W}$ has 24 matchings, 10 of which are stable for $\mathbb{R}_{4}$. Two of them are $\mu_{8}=\left\{\left(m_{1}, w_{3}\right),\left(m_{2}, w_{4}\right),\left(m_{3}, w_{2}\right),\left(m_{4}, w_{1}\right)\right\}$ and $\mu_{9}=\left\{\left(m_{1}, w_{4}\right),\left(m_{2}, w_{3}\right),\left(m_{3}, w_{1}\right),\left(m_{4}, w_{2}\right)\right\}$. $\mu_{8}$ is generated at the third step of the game if the agents of $\left\{w_{4}, w_{3}\right\}$ and $\left\{w_{2}, w_{1}\right\}$ say "yes" and "no", respectively, and the opposite for $\mu_{9}$. And, both for $M$ and $W, \mu_{8}$ and $\mu_{9}$ are incomparable.

Before we state and prove our main theorem, let us examine the following simple example.

Example 12 Let $M=\left\{m_{1}, m_{2}\right\}$ and $W=\left\{w_{1}, w_{2}\right\}$ be the sets of men as the restricter and women as the chooser sides who have the following preference profile $\mathbb{R}_{5}$ :

$$
\mathbb{R}_{5}=\begin{array}{llll}
\frac{m_{1}}{w_{2}} & \frac{m_{2}}{w_{1}} & \frac{w_{1}}{m_{1}} & \frac{w_{2}}{m_{2}} \\
w_{1} & w_{2} & m_{2} & m_{1}
\end{array}
$$

For the sets $M$ and $W$, the set of all the possible matchings is $\Pi^{M \cup W}=$ $\left\{\mu_{1}, \mu_{2}\right\}$ where 


$$
\begin{aligned}
& \mu_{1}=\left\{\left(m_{1}, w_{1}\right),\left(m_{2}, w_{2}\right)\right\}, \\
& \mu_{2}=\left\{\left(m_{1}, w_{2}\right),\left(m_{2}, w_{1}\right)\right\} .
\end{aligned}
$$

For profile $\mathbb{R}_{5}$, the set of stable matchings is $\left\{\mu_{1}, \mu_{2}\right\}$. If $w_{2}$ says "yes" to $m_{1}$, then the best response of $w_{1}$ would be "yes" to $m_{2}$. Otherwise, we get the couples $\mu=\left\{\left(m_{1}, w_{2}\right),\left(m_{2}, m_{2}\right),\left(w_{1}, w_{1}\right)\right\}$. And, if $w_{2}$ says "no" to $m_{1}$, then the best response of $w_{1}$ would be "no" to $m_{2}$; otherwise $w_{1}$ would miss the chance to construct $\left(w_{1}, m_{1}\right)$ which she prefers over $\left(w_{1}, m_{2}\right)$. Hence, the subgame perfect Nash Equilibrium (NE) is $N E\left(w_{1}, w_{2}\right)=\{($ yes, yes $),($ no, no $)\}$. And, it is easy to show that the argument is same for any cyclical conflicts.

Theorem 13 If Nash Equilibria of the cycles are chosen, mechanism $\gamma$ implements the full set of stable matchings for any preference profile. In other words, we always end up with one of the stable matchings for any profile.

Proof. Given our previous results, the proof is simple. Let $\mathbb{R}=\left(R_{i}\right)_{i \in M \cup W}$ be any preference profile with $M$ be the restricter and $W$ be the chooser sides. If there is no cyclical conflict, then there is only one stable matching and our mechanism finds it as we have proved in Theorem 6 .

Hence, let us assume multi stability, and so the existence of some cyclical conflicts. Let $\Pi_{S}^{M \cup W}=\left\{\mu_{1}, \ldots, \mu_{r}\right\}$ be the set of all stable matchings for $\mathbb{R}$. Let us assume $\exists \mu \in \Pi^{M \cup W}$ such that our mechanism does not find it in any scenario.

From Theorem 6 and Proposition 9, we know that $\forall \mu_{x} \in \Pi_{S}^{M \cup W}$ are generated by some cyclical conflicts. Any cycle that has $n$ choosers and $n$ restricters generates $n$ ! matchings; two of them are stable and $(n !-2)$ are unstable. The game ends when all agents construct a pair. With the NE assumption, either all the agents in a cycle say "yes" or all say "no" at the first step of the cycle. As they say "no", we proceed from the top to the bottom on the preferences of the restricters $M$. With the NE assumption, we omit $(n !-2)$ unstable matchings for each cycle which means our mechanism always ends up with a stable matching. So, for each cycle, one of the two stable matchings is chosen.

Hence, if there exists such a matching $\mu$, then either it was not generated by any cycle or it is an unstable matching. If $\mu$ was not generated by any cycle, then from Theorem 6 , it is the unique stable matching for the profile $\mathbb{R}$ which is a contradiction to the assumption on the existence of multi stability. If $\mu$ is unstable, then we are done. Hence, any stable matching could be chosen by our mechanism and there is no possibility to end up with any unstable matching under Nash equilibria.

\subsection{Strategy-Proofness}

Now, we will investigate whether our mechanism is vulnerable to strategic manipulation or not.

Theorem 14 Truth telling (for the choosers) is weakly dominant in our mechanism. 
Proof. Let us assume that our mechanism is manipulable. Let $\mathbb{R}=\left(R_{i}\right)_{i \in M \cup W}$ be any preference profile based on true preferences with $M$ be the restricter and $W$ be the chooser side. And, let $\mathbb{R}^{*}=\left(R_{i}\right)_{i \in M \cup W}$ be any other profile with $R_{i}=R_{i \in M \cup W /\{w\}}^{*}$ and $R_{w} \neq R_{w}^{*}$ for a chooser agent $w \in W . \mathbb{R}^{*}$ is the preference profile based on mispresented preferences and $w$ is the manipulator agent with $\gamma\left(\mathbb{R}^{*}\right) R_{w} \gamma(\mathbb{R})$.

Scenario 1: The preference profile $\mathbb{R}$ has a unique stable matching; that is there is no cyclical conflict. So let $\gamma(\mathbb{R})=\mu$ and $\exists \mu^{*} \in \gamma\left(\mathbb{R}^{*}\right)$. Let $m_{i}, m_{j} \in M$ be two restricters. Let $\mu^{*}(w)=m_{i}$ and $\mu(w)=m_{j}$ with $m_{i} R_{w} m_{j}$. As we have proved, our mechanism $\gamma$ is stable, and so is $\mu^{*}$. To satisfy stability for $\mu^{*}$, we should have $\mu^{*}\left(m_{j}\right) R_{m_{j}} w$ and $w R_{m_{i}} \mu\left(m_{i}\right)$. If we have $\mu^{*}(\bar{m})=\mu(\bar{m})$ for $\forall \bar{m} \in M /\left\{m_{i}, m_{j}\right\}$, then we get $\mu^{*}\left(m_{j}\right)=\mu\left(m_{i}\right)=w^{*}$ such that the sets $\left\{w, w^{*}\right\}$ and $\left\{m_{i}, m_{j}\right\}$ construct a cyclical conflict. If not, to keep the stability of $\mu^{*}$ we should have a pair $\left(\hat{w}, m_{j}\right)$ with $\hat{w} R_{m_{j}} w$, and so on. In every step, we should assign a better mate to every agents which iteratively leads us to the full cyclical conflict. This is a contradiction to the fact that there is no cyclical conflict.

Scenario 2: The preference profile $\mathbb{R}$ has multi stability. If $w$ is not a member of any cycle, then the above argument works. So, we assume that $w$ is a member of a cycle. Let $m_{i}, m_{j} \in M$ be the agents that $w$ experiences the conflict with $m_{i} R_{w} m_{j}$. Let $\mu_{x}$ and $\mu_{y}$ be stable matchings generated from the cycle such that $\mu_{x}(w)=m_{i}$ and $\mu_{y}(w)=m_{j}$. From the definition of a cycle, any agent in the set $\left\{\bar{m} \mid \bar{m} R_{w} m_{i}, \bar{m} \in M\right\}$ is not achievable for $w$. Any matching $\mu^{*}$ such that $m_{j} R_{w} \mu^{*}(w)$ would be unstable. Hence, the question becomes "Can $w$ guarantee to be matched with $m_{i}$ ?".

Firstly, we shall examine Example 12 above, where $\mu_{1}=\mu_{W}$ and $\mu_{2}=\mu_{M}$. Let $w=w_{1}$ be our manipulator. A mispresentation by $w$ breaks the (full) cycle and for new preference profile $\mathbb{R}^{*}$, we get $\gamma\left(\mathbb{R}^{*}\right)=\mu_{2}=\mu_{M}$. Hence, $w=w_{1}$ damages herself by abolishing the possiblity to end up with $\mu_{1}=\mu_{W}$.

Now, we shall consider any cycle with more than two agents for each side. Let $\left\{m_{1}, \ldots, m_{r}\right\} \subset M^{\prime}$ be a set of restricters and $\left\{w_{1}, \ldots, w_{r}\right\} \subset W^{\prime}$ a set of choosers wih $w_{1} R_{m_{1}} w_{2}, w_{2} R_{m_{2}} w_{3}, \ldots, w_{r} R_{m_{r}} w_{1}$ and $m_{1} R_{w_{2}} m_{2}, \ldots, m_{r-1} R_{w_{r}} m_{r}, m_{r} R_{w_{1}} m_{1}$. If any $w_{i} \in W^{\prime}$ changes her preference ordering, she breaks the cycle and saying "yes" to the offers at the first step of the cycle becomes a rational and dominant strategy for every chooser agent in the cycle. And so, while the game could end up with any of the stable matchings $\left\{\mu_{x}, \mu_{y}\right\}$, it ends up with $\mu_{y}$, where $\mu_{x} R_{W^{\prime}} \mu_{y}$ and $\mu_{y}$ and $\mu_{y}$ are the stable matchings generated from the cycle.

Hence, we conclude that truth telling is weakly dominant for the choosers.

\section{The Variant of the Mechanism}

In this section, we introduce a minor change to our mechanism and analyze the effects. We shall call this new mechanism as $\alpha$.

The difference of $\alpha$ from $\gamma$ is that when a chooser agent refuses the offer, 
she is re-placed to the end of the queue of the same restricter agent, instead of loosing him forever as in $\gamma$.

We shall start with a very simple example to analyze the equilibrium.

Example 15 We will focus on $\mathbb{R}_{5}$ in Example 12.

$$
\mathbb{R}_{5}=\begin{array}{llll}
\frac{m_{1}}{w_{2}} & \frac{m_{2}}{w_{1}} & \frac{w_{1}}{m_{1}} & \frac{w_{2}}{m_{2}} \\
w_{1} & w_{2} & m_{2} & m_{1}
\end{array}
$$

For the sets $M$ and $W$, the set of all possible matchings is $\Pi \Pi^{M \cup W}=\left\{\mu_{1}, \mu_{2}\right\}$ where

$$
\begin{aligned}
& \mu_{1}=\left\{\left(m_{1}, w_{1}\right),\left(m_{2}, w_{2}\right)\right\}, \\
& \mu_{2}=\left\{\left(m_{1}, w_{2}\right),\left(m_{2}, w_{1}\right)\right\} .
\end{aligned}
$$

For profile $\mathbb{R}_{5}$, the matchings $\left\{\mu_{1}, \mu_{2}\right\}$ are both stable and for mechanism $\gamma$ the Nash Equilibrium (NE) of this game is $N E\left(w_{1}, w_{2}\right)=\{($ yes, yes $),($ no, no $)\}$. Under mechanism $\alpha$, the story changes.

First, let us assume that $w_{1}$ says "no" and she is replaced to the end of the queue of agent $m_{2}$. At the same step, if $w_{2}$ says "no", then in the second stage of the game, the tentative preference profile will look like:

$$
\mathbb{R}_{5}^{*}=\begin{array}{cccc}
\frac{m_{1}}{w_{1}} & \frac{m_{2}}{w_{2}} & \frac{w_{1}}{m_{1}} & \frac{w_{2}}{m_{2}} \\
w_{2} & w_{1} & m_{2} & m_{1}
\end{array}
$$

Then, in this step both of the choosers say "yes" and we end up with matching $\mu_{1}$, which is chooser-optimal.

On the other hand, if $w_{2}$ says "yes" at the first step, then she forms the pair $\left(m_{1}, w_{2}\right)$ and both of them are deleted from the profile. In the second stage of the game, the tentative preference profile will look like as follows:

$$
\mathbb{R}_{5}^{*}=\frac{m_{2}}{w_{1}}
$$

Then, $w_{1}$ forms the pair $\left(m_{2}, w_{1}\right)$ and we end up with the matching $\mu_{2}$, which is restricter-optimal.

Secondly, let us assume that $w_{1}$ says "yes", then she forms the pair $\left(m_{2}, w_{1}\right)$ and both of them are deleted from the profile. At the first step, whatever $w_{2}$ says, we end up with the matching $\mu_{2}$.

Hence, at the first step, if $w_{1}$ says "yes", the game ends up with $\left\{\mu_{2}\right\}$ and if $w_{1}$ says "no", the game ends up with one of $\left\{\mu_{1}, \mu_{2}\right\}$. So, regardless of which action $w_{2}$ takes, for $w_{1}$ rejecting the offer is weakly dominant. The arguments are the same for $w_{2}$.

Therefore, for mechanism $\alpha$ the Nash Equilibrium (NE) is $N E\left(w_{1}, w_{2}\right)=$ $\{(n o, n o)\}$, which ends up with $\mu_{1}$, the chooser-optimal matching.

We have showed that for profile $\mathbb{R}_{5}, \alpha$ is an improvement for the choosers as compared to mechanism $\gamma$. Of course the opposite is true for the restricters.

We continue with another example.

Example 16 We will study $\mathbb{R}_{2}$ in Example 7. 


$$
\mathbb{R}_{2}=\begin{array}{llllll}
\frac{m_{1}}{w_{1}} & \frac{m_{2}}{w_{2}} & \frac{m_{3}}{w_{2}} & \frac{w_{1}}{m_{3}} & \frac{w_{2}}{m_{1}} & \frac{w_{3}}{m_{3}} \\
w_{3} & w_{1} & w_{3} & m_{2} & m_{2} & m_{1} \\
w_{2} & w_{3} & w_{1} & m_{1} & m_{3} & m_{2}
\end{array}
$$

The preference profile $\mathbb{R}_{2}$ has two stable matchings. The strategic players, having cyclical conflict, are $w_{1}$ and $w_{2}$ in women-chooser case.

The same arguments in Example 15 work here as well. For both $w_{1}$ and $w_{2}$, rejecting the offers at step 1 of the cycle is weakly dominant. Therefore, for mechanism $\alpha$ the Nash Equilibrium (NE) is $N E\left(w_{1}, w_{2}\right)=\{(n o, n o)\}$, which ends up with the chooser-optimal (women optimal) stable matching.

Can we conclude that for all profiles with two stable matchings (one cyclical conflict), $\alpha$ is an improvement for the chooser side as compared to mechanism $\gamma$ ? The following example shows that the answer is negative.

Example 17 Let $M=\left\{m_{1}, m_{2}, m_{3}\right\}$ and $W=\left\{w_{1}, w_{2}, w_{3}\right\}$ be the sets of men and women who have the following preference profile $\mathbb{R}_{6}$ :

$$
\mathbb{R}_{6}=\begin{array}{llllll}
\frac{m_{1}}{w_{1}} & \frac{m_{2}}{w_{2}} & \frac{m_{3}}{w_{1}} & \frac{w_{1}}{m_{2}} & \frac{w_{2}}{m_{1}} & \frac{w_{3}}{m_{1}} \\
w_{3} & w_{1} & w_{3} & m_{1} & m_{3} & m_{2} \\
w_{2} & w_{3} & w_{2} & m_{3} & m_{2} & m_{3}
\end{array}
$$

The preference profile $\mathbb{R}_{6}$ has two stable matchings. The strategic players, having cyclical conflict, are $w_{1}$ and $w_{2}$ in women-chooser case.

First, let us assume that $w_{1}$ accepts the offer and $w_{2}$ does not at step 1 of the cycle. Then, we end up with matching $\mu_{i}=\left\{\left(m_{1}, w_{1}\right),\left(m_{2}, w_{3}\right),\left(m_{3}, w_{2}\right)\right\}$, which $w_{1}$ prefers less than $\mu_{W}=\left\{\left(m_{1}, w_{3}\right),\left(m_{2}, w_{1}\right),\left(m_{3}, w_{2}\right)\right\}$, chooser-optimal matching. So, if $w_{2}$ rejects the offer, so does $w_{1}$.

Second, let us assume that $w_{1}$ rejects the offer and $w_{2}$ accepts at step 1. Then, we end up with $\mu_{j}=\left\{\left(m_{1}, w_{3}\right),\left(m_{2}, w_{2}\right),\left(m_{3}, w_{1}\right)\right\}$, which $w_{1}$ prefers less than $\mu_{M}=\left\{\left(m_{1}, w_{1}\right),\left(m_{2}, w_{2}\right),\left(m_{3}, w_{3}\right)\right\}$, restricter-optimal matching. Therefore, if $w_{2}$ accepts the offer, so does $w_{1}$.

Hence, there is no dominant strategy at step 1. We conclude that $\alpha$ is not an improvement for the choosers for $\mathbb{R}_{6}$.

Our next example is a profile with three stable matchings.

Example 18 Let $M=\left\{m_{1}, m_{2}, m_{3}\right\}$ and $W=\left\{w_{1}, w_{2}, w_{3}\right\}$ be the sets of men and women who have the following preference profile $\mathbb{R}_{7}$ :

$$
\mathbb{R}_{7}=\begin{array}{cccccc}
\frac{m_{1}}{w_{1}} & \frac{m_{2}}{w_{2}} & \frac{m_{3}}{w_{3}} & \frac{w_{1}}{m_{3}} & \frac{w_{2}}{m_{1}} & \frac{w_{3}}{m_{2}} \\
w_{2} & w_{1} & w_{2} & m_{2} & m_{3} & m_{1} \\
w_{3} & w_{3} & w_{1} & m_{1} & m_{2} & m_{3}
\end{array}
$$

$\mathbb{R}_{7}$ has three stable matchings. Now, we will analyze the possible scenarios for $w_{1}$. The table below shows all possible decisions at step 1 and corresponding mate that $w_{1}$ matches from the game. 


$\begin{array}{lllllllll}w_{1} & Y e s & \text { No } & \text { Yes } & \text { No } & \text { Yes } & \text { No } & \text { Yes } & \text { No } \\ w_{2} & Y e s & Y e s & Y e s & Y e s & N o & \text { No } & \text { No } & N_{o} \\ w_{3} & Y e s & Y e s & N o & N o & Y e s & Y e s & N_{o} & N_{o} \\ \text { Mate } & m_{1} & m_{1} & m_{1} & m_{3} & m_{1} & m_{2} & m_{1} & m_{3}\end{array}$

So, we conclude that for $w_{1}$ rejecting the offer at step 1 is weakly dominant. The same analysis for $w_{2}$ and $w_{3}$ shows that also for those agents it is a weakly dominant strategy to refuse. Therefore, for the mechanism $\alpha$ the Nash Equilibrium (NE) is $N E\left(w_{1}, w_{2}, w_{3}\right)=\{(n o, n o, n o)\}$, which ends up with $\mu_{W}=\left\{\left(m_{1}, w_{2}\right),\left(m_{2}, w_{3}\right),\left(m_{3}, w_{1}\right)\right\}$, the chooser-optimal matching.

Example 18 shows that, for $\mathbb{R}_{7}$, which has three stable matchings (the existence of inter-dependent cycles), $\alpha$ is an improvement for the choosers as compared to $\gamma$.

Can we conclude that for all profiles with three stable matchings (interdependent cycles), $\alpha$ is an improvement for the choosers? The following example shows that the answer is negative.

Example 19 We will now study $\mathbb{R}_{3}$.

$$
\mathbb{R}_{3}=\begin{array}{cccccc}
\frac{m_{1}}{w_{1}} & \frac{m_{2}}{w_{2}} & \frac{m_{3}}{w_{2}} & \frac{w_{1}}{m_{3}} & \frac{w_{2}}{m_{1}} & \frac{w_{3}}{m_{2}} \\
w_{2} & w_{1} & w_{3} & m_{2} & m_{2} & m_{1} \\
w_{3} & w_{3} & w_{1} & m_{1} & m_{3} & m_{3}
\end{array}
$$

$\mathbb{R}_{3}$ has three stable matchings. Now, we will analyze the possible scenarios.

First, let us assume that $w_{1}$ accepts the offer and $w_{2}$ does not at step 1 , then we end up with $\mu_{i}=\left\{\left(m_{1}, w_{1}\right),\left(m_{2}, w_{3}\right),\left(m_{3}, w_{2}\right)\right\}$, which $w_{2}$ prefers less than $\mu_{M}=\left\{\left(m_{1}, w_{1}\right),\left(m_{2}, w_{2}\right),\left(m_{3}, w_{3}\right)\right\}$, men-optimal matching. So, if $w_{1}$ accepts the offer, so does $w_{2}$.

Second, let us assume that $w_{1}$ rejects the offer and $w_{2}$ accepts at step 1, then we end up with $\mu_{j}=\left\{\left(m_{1}, w_{3}\right),\left(m_{2}, w_{2}\right),\left(m_{3}, w_{1}\right)\right\}$, which $w_{2}$ prefers less than $\mu_{k}=\left\{\left(m_{1}, w_{2}\right),\left(m_{2}, w_{1}\right),\left(m_{3}, w_{3}\right)\right\}$ (middle stable matching) or $\mu_{W}=$ $\left\{\left(m_{1}, w_{2}\right),\left(m_{2}, w_{3}\right),\left(m_{3}, w_{1}\right)\right\}$ (chooser-optimal matching). Hence, $w_{2}$ is bounded by the action of $w_{1}$ at step 1 .

Finally, let us assume that both of $w_{1}$ and $w_{2}$ reject the offers at step 1 , then we will have the following tentative profile,

$$
\mathbb{R}_{3}^{*}=\begin{array}{cccccc}
\frac{m_{1}}{w_{2}} & \frac{m_{2}}{w_{1}} & \frac{m_{3}}{w_{3}} & \frac{w_{1}}{m_{3}} & \frac{w_{2}}{m_{1}} & \frac{w_{3}}{m_{2}} \\
w_{3} & w_{3} & w_{1} & m_{2} & m_{2} & m_{1} \\
w_{1} & w_{2} & w_{2} & m_{1} & m_{3} & m_{3}
\end{array}
$$

For profile $\mathbb{R}_{3}^{*}$, there are two stable matching $\mu_{W}$ (also women-optimal for $\left.\mathbb{R}_{3}\right)$ and $\mu_{M}\left(\mu_{k}\right.$, middle stable matching for $\left.\mathbb{R}_{3}\right)$. And, it is easy to show that at the first step of $\mathbb{R}_{3}^{*}$ for $w_{1}$ and $w_{3}$, rejecting the offers is weakly dominant which leads us to $\mu_{W}$, the chooser-optimal stable matching.

Since there is no dominant strategy at the first step of $\mathbb{R}_{3}$, we cannot conclude that the outcome set is $\left\{\mu_{M}, \mu_{W}\right\}$ instead of $\left\{\mu_{M}, \mu_{k}, \mu_{W}\right\}$. We will go further 
on the comparisons of those sets since object comparison is not the topic of this paper. But, we regard this shrink as an imrovement for the chooser side.

So far we have showed that for the profiles with independent or interdependent cyclical conflicts, $\alpha$ may or may not be an improvement for the choosers as compared to $\gamma$. Profiles with more than three stable matchings (including incomparable matchings) may have both independent and interdependent cycles. Hence, the same arguments and similar examples, like above, work also for those profiles.

Proposition 20 Let $M=\left\{m_{1}, \ldots, m_{k}\right\}$ be the restricter and $W=\left\{w_{1}, \ldots, w_{l}\right\}$

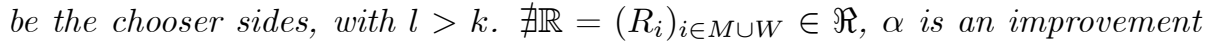
for the choosers; mechanisms $\gamma$ and $\alpha$ coincide.

In plain English, when the number of choosers is more than the number of restricters, mechanism $\alpha$ implements full set of stable matchings as $\gamma$ does.

Proof. For simplicity let us assume $k=n$ and $l=n+1$. Let $\mathbb{R} \in \Re$ be any profile.

If $\mathbb{R}$ has a unique stable matching, then both of $\gamma$ and $\alpha$ finds it. Hence, they coincide.

Let us assume $\mathbb{R}$ has multiple stable matchings. Let $\Pi_{S}^{M \cup W}=\left\{\mu_{1}, \ldots, \mu_{r}\right\}$ be the set of all stable matchings for $\mathbb{R}$. From Roth and Sotomayor (1990), we know that for a profile $\mathbb{R}$, the set of the agents that are matched is the same for all stable matchings. Therefore, the set of $n$ women matched to $n$ men are same for all $\mu \in \Pi^{M \cup W}$.

Let $w^{*} \in W$ be the agent who remains single for all stable matchings. Let $\bar{w} \in W$ be one the paired agents in stable matchings. We only need to show that there exists a cycle where $\bar{w}$ does not have a (weakly) dominant strategy.

Without loss of generality, let us assume $\forall w \in W /\{\bar{w}\}$ accept the offers in the first step of the cycle they confront and $\bar{w}$ rejects the offer by $\bar{m} \in M$ and she is re-placed to the end of the same queue. Since $\forall m \in M /\{\bar{m}\}$ have been taken and deleted from the profile, in the next step $w^{*}$ accepts the offer by $\bar{m} \in M$ and forms the pair $\left(\bar{m}, w^{*}\right) . \bar{w}$ remains single which she prefers less than being matched to $\bar{m}$. Hence, $\bar{w}$ is bounded by the actions of the other agents, like in $\gamma$. The same argument works for any other agent that is matched in stable matchings.

We conclude that $\alpha$ is not an improvement for the chooser side for such preference profiles and it coincides with $\gamma$.

Finally, we state our most general result.

Theorem 21 Let $M=\left\{m_{1}, \ldots, m_{k}\right\}$ be the restricter and $W=\left\{w_{1}, \ldots, w_{l}\right\}$ be the chooser side. Let $\Re$ be the set of all profiles.

Let $\Re^{*}, \Re^{* *}, \Re^{* * *}, \Re^{* * * *} \in \Re$ be disjoint sub-domains such that $\Re^{*} \cup \Re^{* *} \cup$ $\Re^{* * *} \cup \Re^{* * * *}=\Re$, where we have $k \geq l$ for $\Re^{*}, \Re^{* *}$ and $\Re^{* * *}$ and $k<l$ for $\Re^{* * * *}$. 
Let $G S^{C h o o s e r}$ denote the mechanism by Gale and Shapley where, as we call, the chooser side propose.

Let $\theta$ be a mechanism that implements a proper subset of the set of stable matchings, except the chooser-optimal one.

Then, we have

$$
\alpha= \begin{cases}G S^{\text {Chooser }}, & \text { if } \mathbb{R} \in \Re^{*} \\ \theta, & \text { if } \mathbb{R} \in \Re^{* *} \\ \gamma, & \text { if } \mathbb{R} \in \Re^{* * *} \\ \gamma, & \text { if } \mathbb{R} \in \Re^{* * * *}\end{cases}
$$

Proof. Examples $15-19$ and Proposition 20 proves the theorem.

\section{Conclusion}

In this paper, we propose a new dynamic mechanism for semi-centralized two-sided matching games. The model mimics the college admission procedures where the number of agents is too high in the market, like Turkey, Greece, Iran and China, and where the admissions to universities are centralized.

The mechanism is defined on a market where the preferences of the restricter side (the schools) are fixed and we let the chooser side (the students) play the game simultaneously. The outcome matching is determined by the actions of students.

The mechanism partially or fully eliminates the blocking pairs depending on the preference profile under incomplete information. Under complete information, the mechanism implements the full set of stable matchings. Precisely, the set of all possible outcomes of the procedure is the set of stable matchings for any given market.

We also propose a new mechanism, the variant of the original mechanism to improve the outcome of the game for one side (preferably for the students). We show that we are partially successful for this purpose; the variant mechanism is an improvement in a subdomain. Thus, it generates a bias for a preferred side.

Basically the mechanism $\alpha$ partitions the full domain of preference profiles into two subsets. In one of them, it implements a proper subset of stable matchings. And, for some preference profiles, it coincides with $G S^{\text {Chooser }}$, that is it ends up with the chooser-optimal stable matching. In the other, it coincides with $\gamma$, that is it ends up with any of the stable matchings.

We can distinguish those subdomains up to some point. When the number of choosers is more than the restricters, that is $\Re^{* * * *}$, both of the mechanisms coincide. When the number of the choosers is less than or equal to the restricters', we have three subdomains $\Re^{*}, \Re^{* *}$ and $\Re^{* * *}$. While $\alpha$ is an improvementfor $\Re^{*}$ and $\Re^{* *}$, it coincides with $\gamma$ for $\Re^{* * *}$.

Unfortunately, we do not know further about the distinction between $\mathbb{R}^{*}, \mathbb{R}^{* *}$ and $\mathbb{R}^{* * *}$. As we show, the distinction is not based on the cycle types that the preference profiles have. Thus, the outcome of mechanism $\alpha$ is specific to profile. 


\section{References}

[1] Alcalde, J. (1996), "Implementation of Stable Solutions to Marriage Problems", Journal of Economic Theory, 69, 240-254.

[2] Alcalde, J., \& Romero-Medina, A., (2000), "Simple Mechanisms to Implement the Core of the College Admission Problems", Games and Economic Behaviour, 31, 294-302.

[3] Alcalde, J., Pérez-Castrillo, D. \& Romero-Medina, A., (1998), "Hiring Procedures to Implement of Stable Allocations", Journal of Economics Theory, $82,469-480$.

[4] Balinski, M. \& Sönmez, T. (1999), "A Tale of Two Mechanisms: Student Placement", Journal of Economic Theory, 84, 73-94.

[5] Blair, C. (1988), "The Lattice Structure of the Set of Stable Matchings with Multirple Partners", Mathematics of Operations Research, 13(4), 619-628.

[6] Blum, Y., Roth, A.E., \& Rothblum, U.G., (1997), "Vacancy Chains and Equilibration in Senior-level Labour Markets", Journal of Economic Theory, $76,362-411$.

[7] Diamantoudi, E., Miyagawa, E., \& Xue, L., (2007), "Decentralized Matching: the Role of Commitment", Mimeo.

[8] Doğan, K. \& Yuret, D. (2010), "Üniversitelere Öğrenci Yerleştirme Sisteminde Tercih Bildirimindeki Kısıtlamanın Etkileri", Ankara Üniversitesi SBF Dergisi, vol 65, 59-88 (in Turkish).

[9] Gale, D. \& Shapley, L.S. (1962), "College Admissions and the Stability of Marriage", The American Mathematical Monthly, 69: 9-15.

[10] Haeinger, G. \& Wooders, M. (2011), "Decentralized Job Matching", International Journal of Game Theory, vol 40, 1-28.

[11] Knuth, D.E. (1976), "Mariages Stables et leurs relations avec d'autres problèmes combinatoires", Les Presses de l'Université Montréal, Montréal.

[12] Niederle, M. \& Yariv, L., (2009), "Decentralized matching with aligned preferences", Mimeo.

[13] Pais, J., (2008), "Incentives in decentralized random matching markets", Games and Economic Behaviour, 64:632-649.

[14] Peleg, B., (1997), "Implementation the core of a marriage problem", Discussion Paper \#132, Hebrew University of Jerusalem.

[15] Romero-Medina, A., \& Triossi, M., (2013), "Non-revelation mechanisms in many-to-one markets", Games and Economic Behaviour, http://dx.doi.org/10.1016/j.geb.2013.08.005 
[16] Roth, A., E., \& Xing, X., (1997), "Turnaround time and bottlenecks in market clearing: Decentralized matching in the market for clinical psychologists", Journal of Political Economy, 105, 284-329.

[17] Roth,A.E.\&Sotomayor,M. (1990), "Two-Sided Matching: A Study in Game-Theoretic Modelling and Analysis", Econometric Society Monograph Series, Cambridge Univ. Press, New York. 


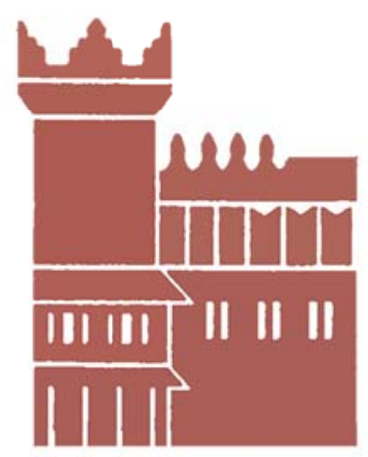

Alma Mater Studiorum - Università di Bologna DEPARTMENT OF ECONOMICS

Strada Maggiore 45

40125 Bologna - Italy

Tel. +39051 2092604

Fax +390512092664

http://www.dse.unibo.it 